\title{
Anxiety Sensitivity and Quality of Life in Patients with Acne
}

\author{
Ersoy Acer, Ali Ercan Altınoz ${ }^{1}$, Hilal Kaya Erdogan, Esra Agaoglu, Nihan Yuksel Canakcı, \\ Zeynep Nurhan Saracoglu
}

Departments of Dermatology and ${ }^{1}$ Psychiatry, Eskisehir Osmangazi University Faculty of Medicine, Eskisehir, Turkey

Background: Anxiety sensitivity (AS) is a continuous fundamental fear and defined as extreme fear of anxiety sensations and symptoms. High AS can cause tendency to anxiety disorders. There are many studies evaluating the anxiety in patients with acne; however, AS has not been investigated. Objective: We aimed to investigate AS in patients with acne. Methods: Two hundred and fourteen acne patients and 117 healthy control subjects, aged older than 16 years were enrolled in the study. Severity of acne was evaluated by Global Acne Grading System. The acne patients and healthy controls were asked to complete the anxiety sensitivity index-3 (ASI-3), the Beck anxiety inventory (BAI) and the Turkish version of acne quality of life index. Results: Anxiety and AS levels in acne patients were significantly higher than healthy controls $(p<0.001)$. Quality of life was impaired in acne patients. Quality of life was negatively correlated with anxiety and AS levels in acne patients $(p=0.014, p=0.019$, respectively). There was no correlation between quality of life and disease severity $(p=0.556)$ and also there was no correlation between psychiatric scale scores (ASI-3, BAI) and disease severity $(p=0.147, p=0.871$, respectively). Conclusion: To our knowledge, our study is the first to report that AS is high in acne patients. Clinically, our results suggest that there

Received August 28, 2018, Revised November 27, 2018, Accepted for publication December 8, 2018

Corresponding author: Ersoy Acer, Department of Dermatology, Eskisehir Osmangazi University Faculty of Medicine, Buyukdere District. Osmangazi University Meselik Campus, 26040 Eskisehir, Turkey. Tel: 90-506-71475-74, Fax: 90-222-239-29-79, E-mail: ersoyacer@hotmail.com ORCID: https://orcid.org/0000-0002-6041-6636

This is an Open Access article distributed under the terms of the Creative Commons Attribution Non-Commercial License (http://creativecommons. org/licenses/by-nc/4.0) which permits unrestricted non-commercial use, distribution, and reproduction in any medium, provided the original work is properly cited.

Copyright (c) The Korean Dermatological Association and The Korean Society for Investigative Dermatology might be a relationship between AS and acne. We conclude that further studies are needed in order to clarify the relationship between acne and AS and to investigate the efficacy of cognitive behavioral treatments in acne patients.

(Ann Dermatol 31(4) $372 \sim 377,2019$ )

\section{-Keywords-}

Acne vulgaris, Anxiety, Anxiety sensitivity, Cognitive therapy, Quality of life

\section{INTRODUCTION}

Acne is a chronic inflammatory disease of pilosebaceous unit. It is a common skin disease affecting approximately $85 \%$ of the adolescents ${ }^{1,2}$. It is characterized by comedones, papules, pustules and/or nodules; finally, these lesions can cause scars ${ }^{1,3}$.

Particularly, facial acne has a considerable psychosocial impact in the affected population. It can cause negative effects on self-image, deterioration in social relations and quality of life and also various psychiatric problems such as anxiety and depression ${ }^{1,4}$. In a recent research, it was reported that $26.2 \%$ of the people with acne had anxiety symptoms. In acne patients; the impairment of quality of life due to acne was found to be associated with severity of the anxiety symptoms ${ }^{5}$.

Anxiety sensitivity (AS) is a continuous fundamental fear and defined as extreme fear of anxiety sensations and symptoms ${ }^{6}$. It is considered as a personality trait and high AS can cause tendency to anxiety disorders (especially panic attack) and alcohol or substance abuse ${ }^{7}$. It is shown that AS: is a distinct entity separate from trait anxiety that can play a role in anxiety and mood disorders and some other psychiatric disorders $^{8-10}$. AS contributes in the mainten- 
ance and exacerbation of a wide variety of chronic physical diseases ${ }^{11}$. Individuals with AS are prone to respond fearfully to anxiety-related sensations. Despite many studies evaluating anxiety frequency in patients with acne, there is lacking literature on AS of the patients with acne. It was reported that patients with psychodermatological diseases like acne or eczema have higher AS than healthy controls $^{12}$. In addition, a recent study showed that AS moderated the association between stress and skin-related emotional and social functioning in adults with dermatological disease ${ }^{13}$.

In this paper we aimed to evaluate the AS in patients with acne. Our hypothesis is that patients with acne have higher AS compared to healthy controls. In addition, the AS scores are negatively correlated with quality of life scores in acne patients.

\section{MATERIALS AND METHODS}

The study was performed between February 2017 and June 2017. Eskisehir Osmangazi University of Local Ethics Committee (decision no: 2017/02) approved the study protocol before the initiation of the study. Acne patients and healthy controls were informed and informed consent form was signed. Each individual participating in the study was informed of the right to withdraw from the study at any time. Participants were selected subsequently from patients with acne who had applied to our department and met the inclusion criteria. For the healthy control group, snowball technique was used for forming the healthy control group which was mostly consisted of hospital personnel. Patients younger than 16 years and with primary neurological disorders, mental retardation, cognitive mental disorders (i.e., dementia and delirium), and psychotic disorders (i.e., schizophrenia, delusional disorder, bipolar affective disorder) were excluded from the study.

Demographic data of the participants were recorded. Severity of acne was evaluated by Global Acne Grading System (GAGS). All volunteers were asked to fill anxiety sensitivity index-3 (ASI-3), Beck anxiety inventory (BAI) and Turkish version of acne quality of life index (TAQLI). Accordingly GAGS, scores are calculated by lesion localizations's factor coefficients (forehead: 2, left cheek: 2, right cheek: 2 , nose: 1 , chin: 1 and neck, chest and back: 3) multiplying with lesion scores (no lesion: 0, comedone: 1, papule: 2, pustule: 3, nodule: 4) and scores of each region are collected. Finally, severity of acne is classified as no ( 0 point), mild ( $1 \sim 18$ points), moderate ( $19 \sim 30$ points), severe (31 $\sim 38$ points) and very severe ( $>39$ points $)^{14}$.

ASI had been defined by Reiss et al. ${ }^{7}$ in 1986 . Later, ASI was revised and a well validated version ASI-3 was developed $^{7,15}$. ASI-3 comprised 3 subscales: physical, cognitive and social. Physical subscale assesses fear of physical symptoms, e.g., be afraid of heart beats rapidly. Cognitive subscale assess fear of losing cognitive control, e.g., be afraid of losing mind. Social subscale assess fear of someone will be aware of their anxiety symptoms, e.g., be afraid of redden in front of society. ASI-3 consists of 18 questions. Each question scored between 0 and 4 . The total score can range between 0 and 72 , the subscale score range between 0 and 24. Higher total scores on this scale suggest higher AS levels and fear of anxiety symptoms. The Turkish form of ASI-3 was shown to be valid and reliable ${ }^{6}$.

$\mathrm{BAI}$ is used to determine the frequency of anxiety symptoms. It includes 21 questions. Each question scored between 0 and 3 . Higher total scores on this scale suggest higher anxiety levels. The validity and reliability of the Turkish version of the BAI was conducted ${ }^{16}$.

TAQLI consists of 11 questions. Each question scored between 0 and 4 . The total score can range between 0 and 44. Higher total scores on this scale suggest more impairment in the quality of life ${ }^{17}$.

IBM SPSS Statistics ver. 21.0 software (IBM Corp., Armonk, NY, USA) was used for the data analyses. Continuous data was presented as mean \pm standard deviation and median (Q1 Q 2 ). Categorical data was presented in percentage and frequency values. Normality was analyzed by Shapiro -Wilk test. Mann-Whitney $U$ test was used to determine the differences between the BAI and ASI scale scores of the two groups and Kruskal-Wallis $\mathrm{H}$ test was used to determine the differences between the BAI, ASI and TAQLI scale scores of the three groups. The Spearman correlation test was used to determine the direction and magnitude of the relationship between the scale scores. $p<0.05$ value was accepted to be statistically significant.

\section{RESULTS}

A total 214 acne patients and 117 healthy controls were enrolled in the study. Mean ages ( \pm standard deviation) in acne and control groups were $21.26 \pm 3.73$ and $21.95 \pm$ 3.39 respectively. One hundred and ten of the acne patients were male, 104 were female; 60 of the healthy controls were male, 57 were female. No significant differences were observed on the age and sex $(p>0.05)$. Mean disease duration ( \pm standard deviation) was $4.27 \pm 3.28$ years (min: $1 \sim \max$ : 18).

Mean ASI-3 total, social, physical subscale, and BAI scores were significantly higher in acne patients compared with controls $(p<0.001, p<0.001, p=0.032, p<0.001$, re- 
spectively) Mean ASI-3 cognitive subscale score was not significantly different between acne patients and controls $(p=0.382)$ (Table 1).

Mean TAQLI score was 16.71 \pm 8.71 (min: 0 max: 40) and mean GAGS score was 14.76 \pm 8.17 (min: $2 \sim \max$ : 38 ) in acne patients. Based on GAGS scores, a total of 150 patients $(70.1 \%)$ had mild, $54(25.2 \%)$ had moderate and $10(4.7 \%)$ had severe acne. The mean ASI-3 total, BAI, and TAQLI scores were not significantly different according to the severity of acne $(p=0.147, p=0.871, p=0.556$, respectively) (Table 2).

In Spearman correlation test, there was significant correlation between TAQLI, BAI and ASI-3 total scores in subjects with acne $(p=0.014, p=0.019$, respectively); but there was no significant correlation between disease duration and BAI and ASI-3 total scores $(p=0.935, p=0.951$, respectively). Similarly, there was no significant correlation between GAGS scores and BAI and ASI-3 total scores ( $p=0.872, p=0.080$, respectively) (Table 3 ).

\section{DISCUSSION}

These results support our hypothesis that patients with acne have higher AS compared to the healthy controls. Specifically, social and physical aspects of AS was found to be significantly higher. These findings suggest that individuals with high AS are more likely to be aware of the social and physical effects of physiological symptoms associated with stress or skin disease. Psychological stress is thought to be related to many common skin diseases and can trigger onset of these diseases or exacerbate ${ }^{18}$. Anxiety can trigger onset of acne or aggravate too. Acne most

Table 1. Comparison of the mean of ASI-3 and BAI scores in acne and control groups

\begin{tabular}{lrrrr}
\hline & Control group $(\mathrm{n}=117)$ & Acne group $(\mathrm{n}=214)$ & $p$-value* \\
\hline ASI-3 social & $4.88 \pm 3.59 / 5.00(2.00 \sim 7.00)$ & $7.93 \pm 4.07 / 8.00(5.00 \sim 10.00)$ & $<0.001$ \\
ASI-3 physical & $4.11 \pm 3.49 / 3.00(1.00 \sim 7.00)$ & $5.18 \pm 4.19 / 4.00(2.00 \sim 8.00)$ & 0.032 \\
ASI-3 cognitive & $4.16 \pm 3.41 / 3.00(1.50 \sim 6.00)$ & $4.83 \pm 4.27 / 4.00(1.00 \sim 7.00)$ & 0.382 \\
ASI-3 total & $13.15 \pm 8.46 / 12.00(6.00 \sim 19.50)$ & $17.94 \pm 10.01 / 17.00(11.00 \sim 23.25)$ & $<0.001$ \\
BAI total & $6.87 \pm 5.04 / 7.00(3.00 \sim 10.00)$ & $11.90 \pm 9.22 / 9.00(5.75 \sim 16.00)$ & $<0.001$ \\
\hline
\end{tabular}

Values are presented as mean \pm standard deviation/median (range). ASI-3: anxiety sensitivity index-3, BAI: Beck anxiety index. ${ }^{*}$ MannWhitney $U$ test.

Table 2. Evaluation of ASI-3, BAI, and TAQLI score averages according to acne severity

\begin{tabular}{lccrl}
\hline & Mild $(\mathrm{n}=150)$ & Moderate $(\mathrm{n}=54)$ & Severe $(\mathrm{n}=10)$ & $p$-value* \\
\hline ASI-3 social & $7.68 \pm 3.94 / 7.00(5.00 \sim 10.00)$ & $8.33 \pm 4.33 / 8.00(5.00 \sim 11.25)$ & $9.60 \pm 4.53 / 9.50(7.00 \sim 13.50)$ & 0.163 \\
ASI-3 physical & $4.85 \pm 3.92 / 4.00(1.75 \sim 7.00)$ & $5.91 \pm 4.74 / 4.50(2.75 \sim 9.00)$ & $6.20 \pm 4.83 / 6.50(1.00 \sim 9.25)$ & 0.304 \\
ASI-3 cognitive & $4.45 \pm 4.06 / 4.00(1.00 \sim 7.00)$ & $5.54 \pm 4.68 / 4.00(2.75 \sim 9.00)$ & $6.80 \pm 4.59 / 7.50(2.50 \sim 10.00)$ & 0.190 \\
ASI-3 total & $16.97 \pm 9.50 / 16.00(10.00 \sim 22.00)$ & $19.78 \pm 11.08 / 18.00(11.00 \sim 26.25)$ & $22.60 \pm 9.66 / 23.50(18.50 \sim 30.25)$ & 0.147 \\
BAI total & $11.78 \pm 9.31 / 9.00(6.00 \sim 15.00)$ & $11.96 \pm 8.53 / 11.00(5.00 \sim 19.00)$ & $13.30 \pm 11.95 / 11.00(3.75 \sim 21.25)$ & 0.871 \\
TAQLI & $16.33 \pm 8.53 / 16.00(10.00 \sim 22.25)$ & $17.54 \pm 9.42 / 16.00(11.00 \sim 23.00)$ & $18.00 \pm 7.82 / 18.00(14.75 \sim 25.25)$ & 0.556 \\
\hline
\end{tabular}

Values are presented as mean \pm standard deviation/median (range). ASI-3: anxiety sensitivity index-3, BAI: Beck anxiety index, TAQLI: Turkish version of acne quality of life index. *Kruskal-Wallis $\mathrm{H}$ test.

Table 3. Correlation between psychometric measurements and the disease duration and GAGS scores

\begin{tabular}{lcccc} 
& \multicolumn{4}{c}{ Correlation coefficient $\left(p\right.$-value $\left.{ }^{*}\right)$} \\
\cline { 2 - 5 } & GAGS & TAQLI & BAI & ASI-3 \\
\hline Duration of disease & $-0.048,0.489$ & $0.008,0.904$ & $0.006,0.935$ & $0.004,0.951$ \\
GAGS & & $0.072,0.294$ & $0.011,0.872$ & $0.120,0.080$ \\
TAQLI & & $0.167,0.014$ & $0.161,0.019$ \\
BAI & & & $0.357,<0.001$ \\
ASI-3 & & & \\
\hline
\end{tabular}

GAGS: Global Acne Grading System, TAQLI: Turkish version of acne quality of life index, BAI: Beck anxiety index, ASI-3: anxiety sensitivity index-3. *Spearman correlation test. 
commonly affects adolescents; that is an important period for physical, social and emotional development. Lower self-confidence, deterioration in social relations and quality of life and also various psychiatric problems have been seen in patients with acne ${ }^{1,4}$. Moreover, psychosocial impact of acne that started in adolescence period can affect whole life of the individual. It has also been reported that acne severity is associated with low social status ${ }^{1}$.

Impairment of quality of life in patients with acne is reported to be similar to in patients with other chronic diseases such as asthma, epilepsy, diabetes, back pain and arthritis $^{19}$. Lukaviciute et al. ${ }^{3}$ reported that quality of life was impaired in $96.5 \%$ of patients with acne. Our results are consistent with these results. Impairment of quality of life is often higher than assessed by the clinician. Severity of acne and its effect on quality of life may not be always correlate. Impairment of quality of life is observed even in patients with mild acne ${ }^{20}$. Similarly, in our study, there was no significant difference in the mean TAQLI scores of patients with acne according to severity of acne $(p=0.556)$. Various psychiatric problems are common in acne patients. In a previous study, it was reported that $56.1 \%$ of the patients with acne had various psychiatric problems. The most common psychiatric problem was anxiety ${ }^{3}$. Golchai et al. $^{21}$ reported that $68.3 \%$, Lukaviciute et al. ${ }^{3}$ $38.4 \%$ of patients with acne had anxiety. In our study, the mean BAI score was significantly higher in acne patients compared with healthy controls. The mean BAI score was higher in severe acne patients than mild-moderate but there was no significant difference. As being a disease that often affects the face and may cause anxiety due to the cosmetic defect in individuals.

There are numerous studies investigating the anxiety frequency in patients with acne; however, AS has never been investigated. Being as a distinct entity separate from trait anxiety, AS is based on the belief that anxiety symptoms have detrimental physical, cognitive, or social consequences. For example, person with high AS may be afraid of sweating, believing that it can be considered a sign of a serious illness or a sign of weakness by others ${ }^{6,8,12}$. It is thought that AS may be associated with various psychodermatological conditions and high AS may increase anxiety responses and severity of dermatological symptoms ${ }^{12,18}$. There are only two studies evaluating AS in dermatological diseases in the literature ${ }^{12,13}$. AS was reported to be significantly higher in psychodermatological diseases (psoriasis, rosacea, acne, etc.) than in non-psychodermatological diseases (sunburn, skin cancer, keloid, etc.) ${ }^{12}$. Similarly, in our study the mean ASI-3 total score was significantly higher in patients with acne and it was higher in patients with severe acne than mild-moderate but there was no significant difference.

TAQLI score was correlated with BAI and ASI-3 total scores in patients with acne but there was no significant correlation between disease duration, GAGS scores, BAI and ASI-3 total scores. These results show that anxiety and AS may be high in patients with acne, regardless of severity and duration of disease and quality of life decreases as anxiety and AS increase. Therefore, even if severity of acne is mild, patients should be evaluated psychologically. A recent study reported that association between stress and skin-related emotional and social functioning in adults with skin disease was moderated by social $\mathrm{AS}^{13}$. Our study reports similar results with the study of Dixon et al. ${ }^{12}$ specially for acne, the main difference is that not only the social AS but also the physical AS was found to be significantly higher. As there are numerous ways for presentation of skin diseases, there might be several psychological mechanisms underneath and authors should evaluate every skin disease in regards to psychological components one by one specifically.

It is important to define the level of AS; because AS can be reduced by short, low-cost interventions ${ }^{22}$. Cognitive behavioral treatments may reduce $\mathrm{AS}^{23}$. Initially, cognitive behavioral treatments were developed for individuals with anxiety disorders; however, it has been reported to be effective in the treatment of pain associated with anxiety and irritable bowel syndrome ${ }^{24,25}$. Dixon et al. ${ }^{12}$ suggested that cognitive behavioral treatments may be beneficial for psychodermatological diseases such as acne. Also, in a previous study, cognitive behavioral therapy is found to be beneficial in patients with acne when added to conventional medical treatments ${ }^{26}$. Reducing the AS levels of acne patients may decrease their anxiety levels and increase their quality of life however further research is needed to examine the efficacy of cognitive behavioral treatments in acne patients.

In our study, we found that patients with acne had impaired quality of life, higher anxiety and higher AS. According to severity of acne, there was no significant difference in TAQLI, BAI and ASI-3 scores. We also found a negative correlation between quality of life and anxiety, AS in patients with acne. However, there was no correlation between disease duration, severity of acne, anxiety, AS and quality of life.

The main limitations of this study were its cross-sectional design and the usage of the self-rating scales. Besides, the selection of the healthy controls from the hospital personnel with snowball technique might be another limitation. To our knowledge, our study is the first to report that AS is high in acne patients. Clinically, our results suggest that there might be a relationship between AS and acne. The 
association between higher AS and acne is not clear. Is AS a cause or a result of acne? isn't exactly known. We conclude that further studies are needed in order to clarify the relationship between acne and AS and to investigate the ef ficacy of cognitive behavioral treatments in acne patients.

\section{ACKNOWLEDGMENT}

Thanks to Muzaffer Bilgin for statistical analysis.

\section{CONFLICTS OF INTEREST}

The authors have nothing to disclose.

\section{ORCID}

Ersoy Acer, https://orcid.org/0000-0002-6041-6636

Ali Ercan Altınoz, https://orcid.org/0000-0003-2233-2105

Hilal Kaya Erdogan, https://orcid.org/0000-0002-8172-1920

Esra Agaoglu, https://orcid.org/0000-0001-8985-6224

Nihan Yuksel Canakcl,

https://orcid.org/0000-0002-3299-0876

Zeynep Nurhan Saracoglu,

https://orcid.org/0000-0002-9116-2288

\section{REFERENCES}

1. Gieler U, Gieler T, Kupfer JP. Acne and quality of life-impact and management. J Eur Acad Dermatol Venereol 2015; 29 Suppl 4:12-14.

2. Picardo M, Eichenfield LF, Tan J. Acne and rosacea. Dermatol Ther (Heidelb) 2017;7(Suppl 1):43-52.

3. Lukaviciute L, Navickas P, Navickas A, Grigaitiene J, Ganceviciene R, Zouboulis CC. Quality of life, anxiety prevalence, depression symptomatology and suicidal ideation among acne patients in Lithuania. J Eur Acad Dermatol Venereol 2017;31:1900-1906.

4. Salman A, Kurt E, Topcuoglu V, Demircay Z. Social anxiety and quality of life in vitiligo and acne patients with facial involvement: a cross-sectional controlled study. Am J Clin Dermatol 2016;17:305-311.

5. Yazici K, Baz K, Yazici AE, Köktürk A, Tot S, Demirseren D, et al. Disease-specific quality of life is associated with anxiety and depression in patients with acne. J Eur Acad Dermatol Venereol 2004;18:435-439.

6. Mantar A, Yemez B, Alkın T. [The validity and reliability of the Turkish version of the anxiety sensitivity index-3]. Turk Psikiyatri Derg 2010;21:225-234. Turkish.

7. Reiss S, Peterson RA, Gursky DM, McNally RJ. Anxiety sensitivity, anxiety frequency and the prediction of fearfulness. Behav Res Ther 1986;24:1-8.

8. McNally RJ. Is anxiety sensitivity distinguishable from trait anxiety? Reply to Lilienfeld, Jacob, and Turner (1989). J Abnorm Psychol 1989;98:193-194.
9. Naragon-Gainey K. Meta-analysis of the relations of anxiety sensitivity to the depressive and anxiety disorders. Psychol Bull 2010;136:128-150.

10. Schmidt NB, Zvolensky MJ, Maner JK. Anxiety sensitivity: prospective prediction of panic attacks and Axis I pathology. J Psychiatr Res 2006;40:691-699.

11. Asmundson GJG, Wright KD, Hadjistavropoulos HD. Anxiety sensitivity and disabling chronic health conditions: state of the art and future directions. Scand J Behav Ther 2000; 29:100-117.

12. Dixon LJ, Lee AA, Viana AG, McCowan NK, Brodell RT, Tull MT. Anxiety sensitivity in dermatological patients. Psychosomatics 2016;57:498-504.

13. Dixon LJ, Witcraft SM, McCowan NK, Brodell RT. Stress and skin disease quality of life: the moderating role of anxiety sensitivity social concerns. Br J Dermatol 2018;178: 951-957.

14. Doshi A, Zaheer A, Stiller MJ. A comparison of current acne grading systems and proposal of a novel system. Int J Dermatol 1997;36:416-418.

15. Taylor S, Zvolensky MJ, Cox BJ, Deacon B, Heimberg RG, Ledley $\mathrm{DR}$, et al. Robust dimensions of anxiety sensitivity: development and initial validation of the Anxiety Sensitivity Index-3. Psychol Assess 2007;19:176-188.

16. Ulusoy $\mathrm{M}$, Sahin $\mathrm{NH}$, Erkmen $\mathrm{H}$. Turkish version of the beck anxiety inventory: psychometric properties. J Cogn Psychother 1998; 12:163-172.

17. Tuncayengin Ö, Gürel MS, ve Şimşek Z. [Development of Turkish quality of life index in acne vulgaris and its changing with acne severity]. Turk Klinikleri J Dermatol 2010;20:117125. Turkish.

18. Orion E, Wolf R. Psychological factors in skin diseases: stress and skin: facts and controversies. Clin Dermatol 2013; 31:707-711.

19. Mallon E, Newton JN, Klassen A, Stewart-Brown SL, Ryan TJ, Finlay AY. The quality of life in acne: a comparison with general medical conditions using generic questionnaires. $\mathrm{Br}$ J Dermatol 1999;140:672-676.

20. Demircay Z, Seckin D, Senol A, Demir F. Patient's perspective: an important issue not to be overlooked in assessing acne severity. Eur J Dermatol 2008;18:181-184.

21. Golchai J, Khani SH, Heidarzadeh A, Eshkevari SS, Alizade $\mathrm{N}$, Eftekhari $\mathrm{H}$. Comparison of anxiety and depression in patients with acne vulgaris and healthy individuals. Indian J Dermatol 2010;55:352-354.

22. Norr AM, Gibby BA, Schmidt NB. Is computerized psychoeducation sufficient to reduce anxiety sensitivity in an atrisk sample?: a randomized trial. J Affect Disord 2017;212: 48-55.

23. Smits JA, Berry AC, Tart CD, Powers MB. The efficacy of cognitive-behavioral interventions for reducing anxiety sensitivity: a meta-analytic review. Behav Res Ther 2008;46: 1047-1054.

24. Craske MG, Wolitzky-Taylor KB, Labus J, Wu S, Frese M, Mayer EA, et al. A cognitive-behavioral treatment for irritable bowel syndrome using interoceptive exposure to visceral sensations. Behav Res Ther 2011;49:413-421. 
25. Watt MC, Stewart SH, Lefaivre MJ, Uman LS. A brief cognitive-behavioral approach to reducing anxiety sensitivity decreases pain-related anxiety. Cogn Behav Ther 2006;35: 248-256.
26. Jung J, Hwang EJ. Do patients with acne need cognitive behavioral therapy? An analysis of patient knowledge and behavior. Int J Dermatol 2012;51:1319-1324. 